\title{
Who ever heard of 16p11.2 deletion syndrome? Parents' perspectives on a susceptibility copy number variation syndrome
}

\author{
Lotte Kleinendorst $^{1,2} \cdot$ Lieke M. van den Heuvel $^{1} \cdot$ Lidewij Henneman $^{2} \cdot$ Mieke M. van Haelst $^{1,2}$
}

Received: 29 October 2019 / Revised: 3 April 2020 / Accepted: 28 April 2020 / Published online: 15 May 2020

(c) The Author(s), under exclusive licence to European Society of Human Genetics 2020

\begin{abstract}
Chromosomal microarray analysis is an important diagnostic tool to identify copy number variations (CNV). Some of the CNVs affect susceptibility regions, which means that deletions or duplications in these regions have partial penetrance and often give an increased risk for a spectrum of neurocognitive disorders. Not much is known about the impact of rare CNV susceptibility syndromes on the life of patients or their parents. In this study, we focus on one specific susceptibility CNV disorder, 16p11.2 deletion syndrome. This rare condition is characterised by an increased risk of mild intellectual disability, autism spectrum disorder, epilepsy, and obesity. We aimed to explore the impact of such a disorder on the family members involved in the daily care of children with this syndrome. Three focus group discussions were held with 23 Dutch (grand) parents. Thematic analysis was performed by two independent researchers. The following five themes emerged: (1) the end of a diagnostic odyssey and response to the diagnosis, (2) after the diagnosis-life with a child with 16p11.2 deletion syndrome, (3) access to medical care and support services, (4) nobody knows what 16p11.2 deletion syndrome is, and (5) future perspective-ideal care. The participants experienced a lack of knowledge among involved professionals. Together with the large variability of the syndrome, this led to fragmented care and unfulfilled needs regarding healthcare, social, and/ or educational assistance. Care for children with a CNV susceptibility syndrome could be improved by a multidisciplinary approach or central healthcare professional, providing education and information for all involved professionals.
\end{abstract}

\section{Introduction}

Over the last decades the possibilities for genetic diagnostics have increased enormously, which enables healthcare professionals to provide a diagnosis for patients with previously unexplained symptoms. A consequence of a larger availability of genetic tests is that more patients and doctors are confronted with findings of uncertain significance or diagnoses with a large phenotypic variability. A

Supplementary information The online version of this article (https:// doi.org/10.1038/s41431-020-0644-6) contains supplementary material, which is available to authorized users.

$\triangle$ Lotte Kleinendorst

1.kleinendorst@amsterdamumc.nl

1 Department of Clinical Genetics, Amsterdam UMC, University of Amsterdam, Amsterdam, The Netherlands

2 Department of Clinical Genetics and Amsterdam Reproduction \& Development research institute, Amsterdam UMC, Vrije Universiteit Amsterdam, Amsterdam, The Netherlands frequently used genetic test that can lead to complex or uncertain findings is chromosomal microarray analysis, which is a first-tier clinical diagnostic test for developmental delay or congenital anomalies [1]. Assessing the pathogenic effects of the copy number variation (CNV), interpreting the results, and communicating them to the patients and parents can be challenging for healthcare providers [2]. This is especially difficult in the case of CNVs that influence the susceptibility of an individual to specific symptoms or diseases. For many of these so-called "susceptibility CNVs", the main symptoms are developmental delay and psychiatric problems. Some people with a susceptibility CNV do not experience any problems at all. Since many of these rare syndromes have only been recently discovered, most of them are still quite unknown. The most studied $\mathrm{CNV}$ is 22q11.2 deletion, which causes the most prevalent CNV syndrome [3]. For 22q11.2 deletion syndrome, a systematic review of studies on the psychosocial impact has shown that parents perceive a lack of knowledge and awareness regarding the syndrome amongst healthcare providers and that they need multidisciplinary care [4]. The increased risk of psychiatric diseases later in childhood 
appears to be especially challenging to discuss with parents of patients with 22q11.2 deletion syndrome [5]. This is also relevant for other CNVs, since many are associated with an increased risk of psychiatric problems [6]. The second most common microdeletion syndrome $16 \mathrm{p} 11.2$ deletion syndrome is much less known and less studied. Little is known about the impact of this syndrome on the life of the patients or their parents, and about their needs and preferences in this regard. Therefore, this qualitative study focused on $16 \mathrm{p} 11.2$ deletion syndrome.

\section{6p11.2 deletion syndrome}

The name " $16 \mathrm{p} 11.2$ deletion syndrome" is used for a variety of microdeletions at the $16 \mathrm{p} 11.2$ region. Most of the times, the "typical 16p11.2" deletion of $\sim 550-600 \mathrm{~kb}$ microdeletion (29.6-30.2 Mb, reference genome GRCh37/hg19) is meant when discussing 16p11.2 deletion syndrome (Online Mendelian Inheritance in Man \#611913). It has been shown that $16 \mathrm{p} 11.2$ deletion was identified in 1 in 235 in a cohort of over 15,000 cases who underwent chromosomal microarray testing [3]. The deletion is most common in subgroups of patients with autism spectrum disorder; it can be found in 1 in 100 children diagnosed with autism [7-9]. The clinical phenotype is variable, with developmental delay and autism as the most frequently observed characteristics. The majority of the patients will experience speech and language deficits [10]. Around 20\% of the patients have epilepsy. Other related medical problems are obesity and vertebral anomalies. Macrocephaly is present in many patients [11]. Although it is an autosomal dominant inherited disorder, most 16p11.2 deletions occur de novo. In the case of an inherited deletion, the clinical phenotype can vary between the affected family members [9]. There are inherited cases where the parent does not show any clinical signs of the 16p11.2 deletion syndrome [7]. In this study, we explored families' perceptions of the impact of a relatively new CNV syndrome, 16p11.2 deletion syndrome. Moreover, we aimed to explore their experiences with healthcare provision and the availability of information. The results of this study will help us to identify the needs of families, which can guide us to pinpoint areas for improvement regarding healthcare and information provision for patients with susceptibility CNVs.

\section{Subjects and methods}

\section{Study design}

We performed three focus groups with parents and other family members involved in daily care of patients with 16 p11.2 deletion syndrome to gain insight into their experiences and perspectives. With the design of focus groups, we expected that this would stimulate a lively discussion among participants and so that we could further explore their different perspectives [12]. The Medical Ethical Committee of the Academic Medical Centre assessed the study protocol and confirmed that the study was exempt from ethics review according to the Medical Research Involving Human Subjects Act (WMO W18-124).

\section{Subjects}

Patients, parents, and other caregivers from all over The Netherlands were invited via the Dutch Facebook group for $16 \mathrm{p} 11.2$ deletion syndrome and the Dutch society for patients with rare genetic disability disorders (ZeldSamen) to register for the $16 \mathrm{p} 11.2$ deletion syndrome information event in 2018. This event has been organised by clinical geneticist $(\mathrm{MMvH})$ and clinical researcher $(\mathrm{LK})$ with the aim to provide the best care for affected individuals by gaining insights in their questions and needs. Based on registration, parents and other family members involved in the daily care of individuals with $16 \mathrm{p} 11.2$ deletion syndrome were invited by e-mail to also participate in the focus group study. The focus group interviews were scheduled on an appropriate date and time for participants. Written informed consent was received from all participants prior to the focus group sessions. All 22 families with a child with $16 \mathrm{p} 11.2$ deletion syndrome who registered for the event were invited to participate in this study, of which 16 families agreed to participate. Of these 16 families, 23 family members were included in the study (Table 1). When multiple family members of one child applied to participate in the study ( $N=6$ family members), they were placed in different focus groups. This led to a maximum of three participating family members per proband to prevent overrepresentation of certain families and their specific experiences. Participants had a median age of 46 years (interquartile range $45-59$ years); $56.5 \%$ of the participants were female. The participants were mostly biological parents (19 biological parents, one foster parent, three grandparents). The median age of the children was 9 years (interquartile range 7.3-12.8). All children had the "typical" 550-600 kb 16p11.2 deletion, as well as four of the participating parents. All children of the participants had developmental delay, intellectual disability, or learning problems to various degrees. All except one child attended special education. Seven children had received a diagnosis for a psychiatric disorder including autism spectrum disorder, attention deficit hyperactivity disorder, and depression. Two children were diagnosed with epilepsy. Table 1 shows further sociodemographic and clinical characteristics of the participants included in the focus groups. 
Table 1 Sociodemographic and clinical characteristics.

\begin{tabular}{|c|c|}
\hline Participants (parents/caregivers) $N=23$ & $N(\%)$ \\
\hline \multicolumn{2}{|l|}{ Gender } \\
\hline Female & $13(56.5)$ \\
\hline Male & $10(43.5)$ \\
\hline \multicolumn{2}{|l|}{ Relation to patient } \\
\hline Biological parent & $19(82.6)$ \\
\hline Foster parent & $1(4.3)$ \\
\hline Grandparent & $3(13.0)$ \\
\hline \multicolumn{2}{|l|}{ Age } \\
\hline $20-30$ & $0(0)$ \\
\hline $30-40$ & $2(8.7)$ \\
\hline $40-50$ & $13(56.5)$ \\
\hline $50-60$ & $4(17.4)$ \\
\hline $60+$ & $4(17.4)$ \\
\hline \multicolumn{2}{|l|}{ Education level $^{\mathrm{a}}$} \\
\hline Low & $6(26.1)$ \\
\hline Moderate & $8(34.8)$ \\
\hline High & $9(39.1)$ \\
\hline \multicolumn{2}{|l|}{ Genetic status of the participant } \\
\hline 16p11.2 deletion & $4(17.4)$ \\
\hline No 16p11.2 deletion & $15(65.2)$ \\
\hline Not tested & $4(17.4)$ \\
\hline Participants' children $N=16$ & $N(\%)$ \\
\hline \multicolumn{2}{|l|}{ Gender } \\
\hline Female & $8(50)$ \\
\hline Male & $8(50)$ \\
\hline \multicolumn{2}{|l|}{ Age } \\
\hline $0-4$ years & $1(6.3)$ \\
\hline $4-8$ years & $3(18.8)$ \\
\hline $8-12$ years & $9(56.3)$ \\
\hline$>12$ years & $3(18.8)$ \\
\hline \multicolumn{2}{|l|}{ Genetic status of the child } \\
\hline Typical 550-600 kb 16p11.2 deletion & $16(100)$ \\
\hline \multicolumn{2}{|l|}{$\mathrm{IQ}^{\mathrm{b}}$} \\
\hline$<70$ & $1(6.3)$ \\
\hline $70-79$ & $2(12.5)$ \\
\hline $80-89$ & $5(31.3)$ \\
\hline $90-109$ & $2(12.5)$ \\
\hline Don’t know & $4(25)$ \\
\hline Never tested & $2(12.5)$ \\
\hline \multicolumn{2}{|l|}{ Psychiatric diagnosis $^{\mathrm{c}}$} \\
\hline No psychiatric diagnosis & $12(75)$ \\
\hline Autism spectrum disorder & $3(18.8)$ \\
\hline ADHD & $3(18.8)$ \\
\hline Depression & $1(6.3)$ \\
\hline \multicolumn{2}{|l|}{ Diagnosed with epilepsy } \\
\hline Yes & $2(12.5)$ \\
\hline No & $14(87.5)$ \\
\hline
\end{tabular}

$A D H D$ attention deficit hyperactivity disorder, $I Q$ intelligence quotient.

a Low: elementary school, lower level secondary school, lower vocational training; Medium: higher level of secondary school, intermediate vocational training; High: higher vocational training, university.

${ }^{\mathrm{b}} \mathrm{IQ}$ groups according the Wechsler Intelligence Scale for Children in which $90-109$ is an average IQ.

${ }^{\mathrm{c}}$ Does not add up to $100 \%$ because of multiple psychiatric diagnoses per patient.

\section{Data collection}

Researchers with expertise in clinical genetics aspects of 16p.11.2 syndrome (LK) and medical psychology (LMvdH) developed a semi-structured topic guide based on the literature and clinical expertise (see Supplemental Material). Topics included the impact of living with a child diagnosed with 16p11.2 deletion syndrome (both on daily life and on psychological and familial functioning) and the experience with and perspectives on healthcare and information provision about 16p11.2 deletion syndrome. Furthermore, participants were asked to complete a short questionnaire on sociodemographic and clinical characteristics directly after attending the focus group. Two out of three focus group sessions were guided by $\mathrm{LMvdH}$ as moderator, and one focus group by LK. Each focus group was monitored by an observant. The focus groups were conducted at the Amsterdam UMC, location AMC, Amsterdam, The Netherlands. The sessions lasted $1-1.5 \mathrm{~h}$.

\section{Data analysis}

The focus group sessions were audio-recorded and transcribed verbatim. Qualitative data analysis was performed using MAXQDA software version 12.2.1 [13]. Thematic analysis was based on the principles of Braun and Clarke [14]. The transcripts were coded by two independent researchers (LK and LMvdH). Any discrepancies between the two researchers were discussed and solved in consensus. The transcripts were read repeatedly to check for consistency between coding analysis and the data. Based on coding analysis, a structure of main and subthemes was created. Data saturation was reached for main themes and most subthemes [14]. Descriptive statistics were used to report participants' characteristics using SPSS version 25 [15].

\section{Results}

Thematic analysis revealed the following five main themes: (1) the end of a diagnostic odyssey and response to the diagnosis, (2) after the diagnosis - life with a child with 16 p11.2 deletion syndrome, (3) access to medical care and support services, (4) nobody knows what $16 \mathrm{p} 11.2$ deletion syndrome is, and (5) future perspective-ideal care. Table 2 shows exemplar participants' quotes per theme to illustrate the results.

\section{Theme 1: The end of a diagnostic odyssey and response to the diagnosis}

Most participants reported that many different doctors had been consulted before the diagnosis $16 \mathrm{p} 11.2$ deletion 
Table 2 Exemplar quotes per theme.

\begin{tabular}{ll}
\hline Quote number & $\begin{array}{l}\text { Focus group (FG), } \\
\text { participant }(\mathrm{P})\end{array}$
\end{tabular}
participant $(\mathrm{P})$

Theme 1: The end of a diagnostic odyssey and response to the diagnosis
$1.1 \quad$ FG2, P4, F1
$1.2 \quad$ FG1, P3, F2
$\begin{array}{ll}1.3 & \text { FG2, P3, F3 } \\ 1.4 & \text { FG2, P1, F4 }\end{array}$
"We were very relieved then [with the diagnosis]. We were visiting doctors for seven years until we knew what it was. So we were sort of relieved, you know, that we were not crazy. That child does have something".
"I have said, for my own sake and for her interest, this is how she is. Yes a special child, and that gives a parent peace".
"He wants it to be fixed, that the gene will be repaired (...). They should find a medicine for it".
"[Child] thinks everyone has a syndrome. (...) She said 'mommy I'm glad that I don't have my brother's syndrome' - Her brother has a terrible morning mood at the moment - 'I'm glad I have $16 \mathrm{p}$ syndrome".

Theme 2: After the diagnosis-life with a child with 16p11.2 deletion syndrome

$2.1 \quad$ FG2, P2, F5

FG2, P1, F4

Theme 3: Access to medical care and support services
3.1
FG1, P7, F9

3.2

FG2, P9, F12

Theme 4: Nobody knows what 16p11.2 deletion syndrome is

$\begin{array}{ll}4.1 & \text { FG2, P5, F13 } \\ 4.2 & \text { FG2, P1/P4/P5, F4/F1/F13 }\end{array}$

"Come on, that [16p11.2 deletion syndrome] is not a nice name for the syndrome. Couldn't they invent something that children can pronounce as well?"

P4: "A girl on the bus has Down [syndrome], she gets everything [support]. But [child] looks nice, is a pretty girl, that's a pitfall". - P1: "I also often wished she had Down [syndrome]. Purely because it would be easier". - P5: "And there are guidelines for it, but for 16p there is nothing".

"Yes, it makes it a very intangible disease and if you look at the impact on our social life... When we go somewhere I always check [the surroundings], and my wife does so as well. We television of the wall so to speak".

"[My child] looks way too pretty. She looks too good, behaves well outside the house, but at home...".

"P3- I say this very honestly, I am happy that we only had one child in our case. Because, I'm very glad with this boy, because it's a very sweet boy. But I, I couldn't have handled a second one, whether or not he would have it [16p11.2 deletion syndrome] or not (...)" - P4: Yes, I recognise that, we have two [children] and the youngest has this. And my partner really wanted a third [child], but I [said] no. Indeed what you said, I can't handle that".

"We once said, we would like to live abroad for a couple of years (...) but the healthcare and schools and support... It is so important to have that (...). So maybe that's the reason not to go".

"It is quite frustrating sometimes when you do not understand your own child".

"He [child] also can't communicate and he doesn't have any friends. For himself, he does have friends, but the friends don't regard him as a friend".

"When there is a birthday, this may sound weird, I can always find her in the kitchen, begging for food like a dog".

P6 "And in school you get complaints. You give so little food to your child? That kid needs more food. Yes, but she cannot [get more food]!".

P2: "Yes but in the old school of [my child] it was the other way around. The teacher called to ask whether we should skip [child] with birthday treats. I found that very sad".

P6: "That is the problem, we are busy with the individual budget again, but you have to go there every year, that is horrible and I hope you can change that". P1:"Yes because it doesn't change. Their chromosomes will never change (...), but every time we have to explain this again".

P1: "Yes the individual budget. The enormous fight (...) I become very frustrated that she constantly has to be tested by people who don't understand what she has". - P2: "Yes you are at the mercy of the whims of bureaucracy".

"I receive no support, no help. They told me my daughter has it [16p11.2 deletion syndrome] in 2015 (...), I asked [the clinical geneticist]: Who I can talk to about the problems we keep running into? Who can help me? - I still know nothing about this".

"We don't need any help, the only thing we have is that school once asked for extra support, but we don't have any other financial help or anything, and that works great".

F $2, \mathrm{P} 1 / \mathrm{P} 4 / \mathrm{P} 5, \mathrm{~F} 4 / \mathrm{F} 1 / \mathrm{F} 1$ 
Table 2 (continued)

\begin{tabular}{lll}
\hline Quote number & $\begin{array}{l}\text { Focus group (FG), } \\
\text { participant (P) }\end{array}$ & Quote \\
\hline 4.3 & FG1, P7, F9 & $\begin{array}{l}\text { P7: "16p, I think they [the teachers] never read it [the information]"- Moderator: Did you give } \\
\text { [the school] the information? - P7: Yes, yes, I told which syndrome she has. Read it, acquaint } \\
\text { yourself with it!". } \\
\text { "We really need to find a balance, I don't expect that he will get it [obesity]. But it could start } \\
\text { from the age of ten - I first heard it would start at age seven, now at age ten - so he could } \\
\text { develop it [obesity], but I don't expect it". } \\
\text { "It is difficult to say what is part of [his] personality and what has to do with the syndrome". }\end{array}$ \\
$\begin{array}{lll}\text { Theme 5: Future perspective-ideal care } \\
\text { FG1, P7, F9 }\end{array}$ & $\begin{array}{l}\text { "What I miss most is someone or an outpatient clinic or something like that, where I can tell } \\
\text { my story to someone who knows what the syndrome is". } \\
\text { "I think it's a shame that you have to figure it all out by yourself, that you can apply for the } \\
\text { individual budget, for a public transport companion pass, you all need to hear that from } \\
\text { someone else who tells you what's possible". }\end{array}$
\end{tabular}

syndrome was eventually established. They expressed that they were relieved that they finally knew what was going on with their child when the diagnosis was established (Table 2, Quote 1.1). One participant said that it gave her some peace to having confirmed that her child was "a special child" because this made it easier to accept the child's behaviour and problems (Table 2, Quote 1.2). The child's reaction on the diagnostic process or on the genetic diagnosis was only discussed by a few participants: one of them mentioned that her child asked whether it would be possible to repair the genetic syndrome because the child did not want to have this syndrome and to be different from other children (Table 2, Quote 1.3). One participant mentioned that her 8-year-old child believed that everyone had some kind of a syndrome (Table 2, Quote 1.4), thus having 16 p11.2 deletion syndrome is not that special or interesting because every single person has "something" that makes him or her unique.

\section{Theme 2: After the diagnosis-life with a child with 16p11.2 deletion syndrome}

\section{Burden on the family}

Medical problems, such as obesity, constipation, frequent ear-nose-throat infections, and sleeping problems, were reported by several participants. Many participants experienced difficulties in looking after their children and provide the best upbringing (Table 2, Quote 2.1). Most participants felt that taking care of their child takes a lot of effort; only two participants disagreed and said that it was comparable to the upbringing of their other unaffected children. One participant said that the daily care is extremely intensive and that he had to watch him constantly. Many participants experienced a lack of satiety in their children, even after eating. Some participants mentioned that they found it hard to set boundaries related to food, because it made their child unhappy. Some participants told that they did not buy certain types of unhealthy food because the child would secretly eat food. A few participants mentioned a discrepancy between the child's behaviour at home and at school (Table 2, Quote 2.2). At school or with family and friends, they behave properly, but at home some children had tantrums or became verbally aggressive towards parents and siblings. These differences in behaviour at home versus at school were hard to understand for the parents. The diagnosis has led to changes in life choices for some participants; some decided not to have another child, another participant decided not to move abroad (Table 2, Quotes 2.3 and 2.4).

\section{Worries about the child's social life and place in society}

Problems in communication were frequently reported by the participants. Most of the participants expressed that it was difficult for them when they were not able to understand their own child (Table 2, Quote 2.5). It was sometimes reported to be difficult in communication with friends or classmates as well (Table 2, Quote 2.6). One participant mentioned that other children sometimes think that his son speaks a foreign language, because they cannot understand him. For several children the communication problems resulted in having no or limited contact with their peers. Some participants also mentioned that their children were frequently unable to participate in regular social or sports activities often because of their fatigue and communication problems. Their increased appetite also led to problems at school for some children. There was a teacher who thought that the child did not receive enough food (even though the child had obesity); whereas another teacher was worried about the child's obesity and suggested not to give birthday treats to the affected child any longer (Table 2, Quotes 2.7 
and 2.8). Participants reported that school days were generally too long for children, due to large travel distances between home and special education services. Travelling to school requested additional support from some participants. Many children were generally not able to travel to school alone, in contrast to what teachers at school sometimes expected. For other children, no arrangements for travelling to school were available. Multiple participants worried about the ability of their child to do things independently and to live on their own in the future.

\section{Administrative and financial burden}

Many participants experienced the financial and administrative issues related to the support of their child as a burden. The awareness of financial support and the possibilities for support available per child was different between the municipalities where the participants lived in. In The Netherlands, most of these children are eligible for the "individual budget" (budget provided by the government that parents can apply for to arrange and purchase assistance for their child). To receive this budget, the participants often had to prove that their child still needed this additional support. Participants found this frustrating, since once a diagnosis is established, this does not change over time (Table 2, Quote 2.9). Moreover, they felt that the officials judging the application did not understand what the 16p11.2 deletion syndrome exactly means (Table 2, Quote 2.10). Some participants mentioned that they did not receive any financial support at all for their child's healthcare and support. This lack of financial support surprised another participant, because her municipality even paid for professional help to teach her child how to ride a bicycle.

\section{Theme 3: Access to medical care and support services}

The participants mentioned that many different healthcare professionals are involved in the treatment or care of their children. For example, a majority of the patients visits or visited a speech therapist. Many patients also visited a dietician. The majority of participants experienced limited access to support or care. There were few participants who reported that their child received no medical care or support at all (Table 2, Quote 3.1). There was only one participant who told that there was no need for any treatment or support at that time (Table 2, Quote 3.2). Others put much effort and time into requesting care but did not always manage to receive it. These participants were unsure why they did not receive the requested care, but argued that it could be related to the unfamiliarity and variability of the syndrome.

\section{Theme 4: Nobody knows what 16p11.2 deletion syndrome is}

\section{Having a well-known syndrome would be easier}

One participant told it was unclear for them and their children what 16p11.2 deletion syndrome meant and that the name of the syndrome was too difficult for them (Table 2, Quote 4.1). Another participant mentioned that their child did not look different from unaffected children. As a result, other people, including professionals, generally do not understand that these children need additional care and support. Since 16p11.2 deletion syndrome is a rare syndrome that has been recently discovered, many participants felt that the syndrome was relatively unknown, and not all symptoms and characteristics of this condition were clear to healthcare professionals. Participants therefore believed they had to sort out many things themselves, and that it was unclear where to get support or that support was not adequately provided. Some participants therefore believed that it would have made life easier if their child would have been diagnosed with Down syndrome, which was considered a better-known syndrome, instead of 16p11.2 deletion syndrome (Table 2, Quote 4.2). One participant had experienced a lack of knowledge about 16p11.2 deletion syndrome among all involved healthcare providers. More participants observed this lack of knowledge amongst teachers and education professionals involved in the care of their children. Some participants considered teachers incapable of educating this "type" of children because they did not understand what 16p11.2 deletion syndrome involved and how symptoms should be handled and had not read the information about the syndrome that the participant had given them (Table 2, Quote 4.3). However, others were very satisfied with the school and the teachers. Many participants said that their information needs were unmet. Some participants knew about online disorder information, such as the Unique guides or Facebook pages, to find more information about the syndrome. Others were unaware of the available information or desired more information than available. They also expressed the need to share experiences with other parents or caregivers. Information specifically for affected children themselves was perceived as still lacking.

\section{6p11.2 deletion syndrome is variable which leads to uncertainty}

Many participants reported that the variability in symptoms and related consequences of $16 \mathrm{p} 11.2$ deletion syndrome caused uncertainty. The participants discussed that they had to monitor themselves whether or not symptoms were developing in their child, for example whether their child was gaining weight (Table 2, Quote 4.4). Another 
participant mentioned that the variability of behavioural and psychiatric problems as part of $16 \mathrm{p} 11.2$ deletion syndrome was difficult to interpret: the participant was uncertain whether his child's behaviour was related to the syndrome or just his personality (Table 2, Quote 4.5). The variability and uncertainty regarding the intellectual capacity of children with 16p11.2 deletion syndrome was discussed as well. For some children regular education was suitable, while for others special education was needed. Participants with younger children expressed the need for guidance regarding the most suitable school (type) for their child.

\section{Theme 5: Future perspective-ideal care}

After hearing the genetic diagnosis it still remained difficult for some of the participants to receive adequate healthcare and information. For others, the diagnosis had opened doors to appropriate healthcare. Participants mentioned that they would feel supported if there would be personalised or standardised treatment available to relieve the symptoms associated with 16p11.2 deletion syndrome. Some participants noted that it would be better if there was a main healthcare provider to coordinate care or a central place where they could ask questions regarding the syndrome, for example a specialised outpatient clinic (Table 2, Quote 5.1). Two participants mentioned that they would prefer to hear more from doctors and researchers about the experiences with medication in patients with $16 \mathrm{p} 11.2$ deletion syndrome. The participants also wanted to ask non-medical questions to these $16 \mathrm{p} 11.2$ deletion syndrome experts, for example which school type is most suitable for their child. Several participants mentioned that professional assistance in applying for financial support would be helpful as well (Table 2, Quote 5.2).

\section{Discussion}

This focus group study gave insight in the perspectives and experiences of (grand)parents on having a child with 16p11.2 deletion syndrome and the impact of this particular CNV syndrome on their child's and family's lives. Our participants reported that their children experienced many medical and psychosocial problems impacting daily life, and described the challenges for parents raising their children. The stories of the participants uncovered a variety of important themes that, to our knowledge, have not been discussed in the literature before in the context of 16p11.2 deletions or similar susceptibility CNVs.

Our participants felt relieved once the diagnosis for their child was established. This "end of a diagnostic odyssey" is a well-known term in the genetic literature [16]. Receiving a genetic diagnosis can be very helpful for parents, since this will give insights in the symptoms or intellectual development they can expect for their child, and comorbidities associated with the disease can be monitored. The benefits of knowing the genetic diagnosis were mentioned by our participants, but appeared to be less evident for them in the long term. The unpredictability whether their child will have learning problems or develop symptoms such as obesity is difficult to handle for parents. In fact, experiencing uncertainty following the diagnosis is a common problem with CNVs $[17,18]$. This uncertainty has multiple causes: there is often incomplete penetrance of the phenotype for many CNVs, there is often a large variability of symptoms, and there is (still) a lack of information about the prognosis of the disorder [3, 19]. Moreover, uncertainties about the future are frequently reported by caregivers of patients with chronic diseases in general [19]. Another extensively discussed topic during the focus group meetings was the financial and administrative burden that the parents experience. These findings are in line with the literature on the experiences that parents of children with rare diseases in general have, feeling burdened by their role as care coordinator [20].

Our study also aimed to explore the experiences of parents with the healthcare and information provision. Many of the participants in the current study did not feel sufficiently supported by healthcare providers to learn about the disorder and its associated symptoms. Parents participating in the current study experienced that they were the 16 p11.2 deletion syndrome experts instead of the doctors, especially since many believed that the healthcare providers were unable to give them sufficient information about the syndrome. This is in line with the literature about parents of children with rare diseases, who feel that they are the "expert caregivers" and know more about the disorder than the involved medical specialist [20]. It is possible that the parents did receive adequate counselling about the disorder, but that the parents were not able to obtain the information at that moment. Timing in the delivery of information is therefore important. Moreover, the parents' questions and information needs can change during the development of the child. Repeat healthcare visits to gain information about the disorder during childhood and adolescence can be helpful to address these questions. Although certain information is available for parents, not all participants in our focus group were aware of this. For example, charity organisation Unique offers disorder guides for many rare chromosome disorders to inform both parents and healthcare professionals (www.rarechromo.org). Some participants mentioned that the difficult name of $16 \mathrm{p} 11.2$ deletion syndrome is not beneficial for the general knowledge and awareness of this genetic condition. This will apply to many other deletion or duplication syndromes as well. Parents' wish for a simpler name is important to note because of the 
trend to name genetic disorders on a description of the disorder and the underlying genetic cause [21].

The medical problems associated with 16p11.2 deletion syndrome are diverse and participants mentioned that many different healthcare providers were involved in the treatment of their children. Moreover, they experienced that current healthcare and support is very fragmented. This made them feel that access to care and adequate treatment was restricted. A large literature review of parents that have a child with a chronic disease showed that these parents also experienced difficulties in obtaining information and were unsatisfied with the information provided by healthcare professionals [22]. Indeed, many medical professionals acknowledge that organizing medical care for patients with very rare disorders is challenging and needs to be arranged in centres of expertise [23]. Having one main healthcare provider or coordinator of a multidisciplinary team could improve the medical support for children with CNV syndromes and their parents, in which the central healthcare professional provides information about the syndrome to all involved professionals. We think that the input of parents, expressing their concerns and wishes, is necessary to shape this multidisciplinary healthcare team. This thought is supported by previous findings that parents of children with rare diseases find it important that healthcare providers stimulate them in their active participation regarding their child's healthcare [19]. Healthcare providers should also stimulate peer support for these parents. Because of the rarity of these syndromes, it can be difficult for parents to find other parents who are facing similar problems. We would recommend doctors to stimulate parents in visiting patient information events or to refer to disorder support groups on social media.

This study gives new insights in the perspectives of parents regarding 16p11.2 deletion syndrome and microdeletion/duplication susceptibility syndromes in general. A limitation of this study is that we could only perform three focus group interviews because of the relative rarity of the syndrome. However, data saturation was reached for most themes and subthemes. Moreover, the fact that our participants visited a patient information evening and wanted to participate in this study might have led to a bias because they either experienced more problems regarding the syndrome or because they were more interested in patient advocacy and parent support. Our described group of children seems to be representative for patients with $16 \mathrm{p} 11.2$ deletion syndrome in terms of IQ and other characteristics of $16 \mathrm{p} 11.2$ deletion syndrome, although this is difficult to assess with small numbers. However, in our patient group most children did not have a psychiatric diagnosis, whereas the literature reports that a majority of individuals with a 16p11.2 deletion have one or more psychiatric diagnoses.
This qualitative study is the first to explore the experiences and perspectives of parents with a child with 16p11.2 deletion syndrome. The findings of this study offer interesting opportunities for future research. One of our findings that is quite specific for $16 \mathrm{p} 11.2$ deletion syndrome is that some parents reported they needed more support to cope with their child's increased appetite and food behaviour problems. It would be useful to explore this topic further in the future, possibly by learning from other genetic disorders with increased appetite such as Prader-Willi syndrome. Furthermore, research focusing on improving the coordination of care for these families would be recommended. Although 16p11.2 deletion syndrome is relatively rare and the numbers to include are therefore expected to be relatively small, a survey study to assess parents' experiences and psychological impact of having a child with 16p syndrome, incorporating the findings of the current study, would be interesting to further investigate and quantify our findings on group level. In a future study, we also aim to explore the perceptions of patients themselves as well, although this might be difficult to arrange because of the large variability in age and intellectual abilities.

In conclusion, parents reported a lack of understanding and information regarding the syndrome and its associated variability amongst both healthcare providers and other involved professionals, which-for many parents-resulted in fragmented care and support. The 16p11.2 deletion syndrome is quite recently discovered and still unknown to many doctors, even though it is the second most common microdeletion syndrome. Because of new and more commonly used diagnostic genetic techniques, novel syndromes are discovered on a regular basis. It is therefore of vital importance that clinical geneticists and genetic counsellors assess the information needs regarding these syndromes to adequately inform both patients and other involved professionals. The presented findings might be applicable for other susceptibility CNV syndromes as well. This can be used to improve our healthcare for patients with these syndromes and their families. Based on the findings we make the following recommendations for clinical practice: (1) Many participants experienced a lack of information about 16p11.2 deletion syndrome. Repeat visits can be useful during childhood and adolescence to address new questions and needs. This also enables the healthcare provider to divide the extensive information over multiple visits and to repeat provided information, taking the age and developmental phase of the patient into account; (2) doctors can also help to improve information provision by creating awareness of the available guides for rare genetic disorders and other ways to receive up-to-date information such as organising patient information evenings or participating in online support groups for parents; and (3) because of the large variability of symptoms in $16 \mathrm{p} 11.2$ deletion 
syndrome, patients are often treated by many different healthcare providers. A personalised and centralized multidisciplinary approach in both medical treatment as well as psychosocial and educational support is therefore needed.

Acknowledgements We would like to thank all the participants of the focus group interviews. We thank Saskia Kleinendorst for the transcriptions and Mellody Cooiman for her help during the focus group sessions.

\section{Compliance with ethical standards}

Conflict of interest The authors declare that they have no conflict of interest.

Publisher's note Springer Nature remains neutral with regard to jurisdictional claims in published maps and institutional affiliations.

\section{References}

1. Miller DT, Adam MP, Aradhya S, Biesecker LG, Brothman AR, Carter NP, et al. Consensus statement: chromosomal microarray is a first-tier clinical diagnostic test for individuals with developmental disabilities or congenital anomalies. Am J Hum Genet. 2010;86:749-64.

2. Paul JL, Pope-Couston R, Wake S, Burgess T, Tan TY. Communicating microarray results of uncertain clinical significance in consultation summary letters and implications for practice. Eur J Hum Genet. 2016;25:22-30.

3. Kaminsky EB, Kaul V, Paschall J, Church DM, Bunke B, Kunig $\mathrm{D}$, et al. An evidence-based approach to establish the functional and clinical significance of copy number variants in intellectual and developmental disabilities. Genet Med. 2011;13:777-84.

4. Vo OK, McNeill A, Vogt KS. The psychosocial impact of 22q11 deletion syndrome on patients and families: a systematic review. Am J Med Genet A. 2018;176:2215-25.

5. Martin N, Mikhaelian M, Cytrynbaum C, Shuman C, Chitayat DA, Weksberg R, et al. 22q11.2 deletion syndrome: attitudes towards disclosing the risk of psychiatric illness. J Genet Couns. 2012;21:825-34.

6. Thapar A, Cooper M. Copy number variation: what is it and what has it told us about child psychiatric disorders? J Am Acad Child Adolesc Psychiatry. 2013;52:772-4.

7. Bijlsma EK, Gijsbers AC, Schuurs-Hoeijmakers JH, van Haeringen A, Fransen van de Putte DE, Anderlid BM, et al. Extending the phenotype of recurrent rearrangements of $16 \mathrm{p} 11.2$ : deletions in mentally retarded patients without autism and in normal individuals. Eur J Med Genet. 2009;52:77-87.

8. Stefansson H, Meyer-Lindenberg A, Steinberg S, Magnusdottir B, Morgen K, Arnarsdottir S, et al. CNVs conferring risk of autism or schizophrenia affect cognition in controls. Nature. 2014;505: 361-6.

9. Weiss LA, Shen Y, Korn JM, Arking DE, Miller DT, Fossdal R, et al. Association between microdeletion and microduplication at 16p11.2 and autism. N Engl J Med. 2008;358:667-75.

10. Mei C, Fedorenko E, Amor DJ, Boys A, Hoeflin C, Carew P, et al. Deep phenotyping of speech and language skills in individuals with 16p11.2 deletion. Eur J Hum Genet. 2018;26:676-86.

11. Miller DT, Chung W, Nasir R, Shen Y, Steinman KJ, Wu BL, et al. $16 \mathrm{p} 11.2$ recurrent microdeletion. In: Adam MP, Ardinger HH, Pagon RA, Wallace SE, Bean LJH, Stephens K, et al., editors. GeneReviews((R)). Seattle, WA; 1993.

12. Kitzinger J. Qualitative research. Introducing focus groups. BMJ. 1995;311:299-302.

13. VERBI Software. MAXQDA analytics Pro 12 ed. Berlin (Germany): VERBI; 2017.

14. Braun V, Clarke V. Using thematic analysis in psychology. Qualitative Res Psychol. 2006;3:77-101.

15. IBM Corporation. SPSS statistics for Windows, version 24.0. Armonk, NY: IBM Corporation; 2016.

16. Rosenthal ET, Biesecker LG, Biesecker BB. Parental attitudes toward a diagnosis in children with unidentified multiple congenital anomaly syndromes. Am J Med Genet. 2001;103:106-14.

17. Reiff M, Bernhardt BA, Mulchandani S, Soucier D, Cornell D, Pyeritz RE, et al. "What does it mean?": uncertainties in understanding results of chromosomal microarray testing. Genet Med. 2012;14:250-8.

18. Reiff M, Ross K, Mulchandani S, Propert KJ, Pyeritz RE, Spinner NB, et al. Physicians' perspectives on the uncertainties and implications of chromosomal microarray testing of children and families. Clin Genet. 2013;83:23-30.

19. Cardinali P, Migliorini L, Rania N. The caregiving experiences of fathers and mothers of children with rare diseases in italy: challenges and social support perceptions. Front Psychol. 2019;10:1780.

20. Baumbusch J, Mayer S, Sloan-Yip I. Alone in a Crowd? Parents of children with rare diseases' experiences of navigating the healthcare system. J Genet Couns. 2019;28:80-90.

21. Turnpenny P, Smith R. Of eponyms, acronyms and...orthonyms. Nat Rev Genet. 2003;4:152-6.

22. Smith J, Cheater F, Bekker H. Parents' experiences of living with a child with a long-term condition: a rapid structured review of the literature. Health Expect. 2015;18:452-74.

23. Hennekam RC. Care for patients with ultra-rare disorders. Eur $\mathbf{J}$ Med Genet. 2011;54:220-4. 